\title{
Existence results for nonlinear quadratic functional integral equations of fractional orders
}

\author{
A. M. A. El-Sayed and H. H. G. Hashem
}




\title{
EXISTENCE RESULTS FOR NONLINEAR QUADRATIC FUNCTIONAL INTEGRAL EQUATIONS OF FRACTIONAL ORDERS
}

\author{
A. M. A. EL-SAYED AND H. H. G. HASHEM
}

Received 18 September, 2012

\begin{abstract}
We present an existence theorem for at least one continuous solution for a nonlinear quadratic functional integral equation of fractional (arbitrary) order. Also, we prove the existence of maximal and minimal solutions for that equations.
\end{abstract}

2000 Mathematics Subject Classification: 32A55; 11D09

Keywords: quadratic integral equation of fractional-order, Schauder fixed point theorem, continuous solution, maximal and minimal solutions

\section{INTRODUCTION}

It is well known that integral equations have many useful applications in describing numerous events and problems of real world. Moreover the theory of integral equations is rapidly developing using the tools of functional analysis, topology and fixed point theory. In particular, quadratic integral equations have many useful applications in the real world. For example, quadratic integral equations are often applicable in the theory of radiative transfer, the kinetic theory of gases, the theory of neutron transport, queuing theory and traffic theory. Many authors studied the existence of solutions for several classes of nonlinear quadratic integral equations (see e.g. [1-11] and $[8-17,19]$. However, in most of the above literature, the main results are realized with the help of the technique associated with the measure of noncompactness. Instead of using the technique of measure of noncompactness we use Schauder fixed point theorem.

Let $\mathbb{R}$ be the set of real numbers whereas $I=[0,1], L_{1}=L_{1}[0,1]$ be the space of Lebesgue integrable functions on $I$. 
Here, we prove the existence of at least one continuous solution of the quadratic functional integral equation of fractional order

$$
x(t)=a(t)+g(t, x(\psi(t))) \int_{0}^{t} \frac{(t-s)^{\alpha-1}}{\Gamma(\alpha)} f(s, x(\phi(s))) d s, \quad t \in I, \quad \alpha>0
$$

and the existence of a continuous solution of the nonlinear functional differential equation of fractional-order

$$
{ }_{R} D^{\alpha} x(t)=f(t, x(\phi(t))), t \in I \text { and } x(0)=0, \quad \alpha \in(0,1)
$$

(where ${ }_{R} D^{\alpha}$ is the Riemann-Liouville fractional order derivative) will be given as an application. Also the results concerning the existence of continuous solution of the initial value problem

$$
\frac{d x(t)}{d t}=f(t, x(\phi(t))), \quad x(0)=x_{0},
$$

will be given as another application. Finally, the existence of maximal and minimal solutions of (1.1) will be proved.

For $\psi(t)=\phi(t)=t$, J. Banas and B. Rzepka( see [8]) proved the existence of a nondecreasing continuous solution of (1.1) by using the technique of measure of noncompactness. The existence of continuous solutions for some quadratic integral equations was proved by using Schauder-Tychonoff fixed point theorem [25].

\section{PReliminaries AND Definitions}

The existence results will be based on the following fixed-point theorems and definitions.

Theorem 1 (Schauder Fixed Point Theorem in [9]). Let $Q$ be a nonempty, convex, compact subset of a Banach space $X$, and $T: Q \rightarrow Q$ be a continuous map. Then $T$ has at least one fixed point in $Q$.

We shall collect the definitions of the fractional-order integral and differential operators. Let $\beta$ be a positive real number

Definition 1. The fractional-order integral of order $\beta$ of the function $f$ is defined on $[a, b]$ by (see [20], [23], [22] and [26])

$$
I_{a}^{\beta} f(t)=\int_{a}^{t} \frac{(t-s)^{\beta-1}}{\Gamma(\beta)} f(s) d s
$$

and when $a=0$, we have $I^{\beta} f(t)=I_{0}^{\beta} f(t)$.

Definition 2. The Riemann-Liouville fractional-order derivative of order $\beta \in(0,1)$ of the function $f$ is given by (see [20], [23], [22] and [26])

$$
{ }_{R} D^{\beta} f(t)=\frac{d}{d t} I^{1-\beta} f(t) .
$$


For the properties of fractional calculus see [20], [23], [22] and [26] for example.

\section{EXISTENCE OF CONTINUOUS SOLUTIONS}

Now, equation (1.1) will be investigated under the assumptions:

(i) $a: I \rightarrow \mathbb{R}$ is continuous and bound with $k_{1}=\sup _{t \in I}|a(t)|$.

(ii) $g: I \times \mathbb{R} \rightarrow \mathbb{R}$ is continuous and bounded with $k_{2}=\sup _{(t, x) \in I \times \mathbb{R}}|g(t, x)|$.

(iii) There exist two constants $l_{i}, i=1,2$ respectively satisfying

$$
|g(t, x)-g(s, y)| \leq l_{1}|t-s|+l_{2}|x-y|
$$

for all $t, s \in I$ and $x, y \in \mathbb{R}$.

(iv) $f: I \times \mathbb{R} \rightarrow \mathbb{R}$ satisfies Carathèodory condition (i.e. measurable in $t$ for all $x: I \rightarrow \mathbb{R}$ and continuous in $x$ for all $t \in I$ ).

(v) There exist a function $m \in L_{1}$ and a constant $b$ such that $|f(t, x)| \leq m(t)+b|x|(\forall(t, x) \in I \times \mathbb{R})$ and $k_{3}=\sup _{t \in I} I^{\beta} m(t)$ for any $\beta \leq \alpha$.

(vi) $\psi, \phi: I \rightarrow I$ are continuous .

(vii) The inequality $k_{2} b<\Gamma(1+\alpha)$ is satisfied.

Theorem 2. Let the assumptions (i)-(vii) be satisfied. Then the quadratic functional integral equation (1.1) has at least one solution in the space $x \in C(I)$.

Proof. Let $C=C(I)$ be the Banach space of all real functions defined and continuous on the interval $I$.

Fix a number $r>0$ and consider the ball $S_{r}$ in the space $C(I)$ defined as

$$
S_{r}=\{x \in C(I):|x(t)| \leq r \text { for } t \in I\} .
$$

Let $T$ be the operator defined on $S_{r}$ by the formula

$$
(T x)(t)=a(t)+g(t, x(\psi(t))) \int_{0}^{t} \frac{(t-s)^{\alpha-1}}{\Gamma(\alpha)} f(s, x(\phi(s))) d s, \quad x \in S_{r}, t \in I .
$$

Then, in view of our assumptions, for $x \in S_{r}$ and $t \in I$ we get

$$
\begin{gathered}
|T x(t)| \leq|a(t)|+|g(t, x(\psi(t)))| \int_{0}^{t} \frac{(t-s)^{\alpha-1}}{\Gamma(\alpha)}|f(s, x(\phi(s)))| d s \\
\leq k_{1}+k_{2} I^{\alpha-\beta} I^{\beta} m(t)+k_{2} b \int_{0}^{t} \frac{(t-s)^{\alpha-1}}{\Gamma(\alpha)}|x(\phi(s))| d s \\
\leq k_{1}+k_{2} k_{3} \int_{0}^{t} \frac{(t-s)^{\alpha-\beta-1}}{\Gamma(\alpha-\beta)} d s+k_{2} b r \int_{0}^{t} \frac{(t-s)^{\alpha-1}}{\Gamma(\alpha)} d s \\
\leq k_{1}+\frac{k_{2} k_{3}}{\Gamma(\alpha-\beta+1)}+\frac{k_{2} b r}{\Gamma(1+\alpha)} .
\end{gathered}
$$


Hence, in view of assumption (vii) we have that $T$ transforms the ball $S_{r}$ into itself for

$$
r=\left(k_{1}+\frac{k_{2} k_{3}}{\Gamma(\alpha-\beta+1)}\right)\left(1-\frac{k_{2} b}{\Gamma(1+\alpha)}\right)^{-1} .
$$

Now, for $t_{1}$ and $t_{2} \in I$ (without loss of generality assume that $t_{1}<t_{2}$ ), we have

$$
\begin{aligned}
& (T x)\left(t_{2}\right)-(T x)\left(t_{1}\right)=a\left(t_{2}\right)-a\left(t_{1}\right) \\
+ & g\left(t_{2}, x\left(\psi\left(t_{2}\right)\right)\right) I^{\alpha} f\left(t_{2}, x\left(\phi\left(t_{2}\right)\right)\right)-g\left(t_{1}, x\left(\psi\left(t_{1}\right)\right)\right) I^{\alpha} f\left(t_{1}, x\left(\phi\left(t_{1}\right)\right)\right) \\
+ & g\left(t_{1}, x\left(\psi\left(t_{1}\right)\right)\right) I^{\alpha} f\left(t_{2}, x\left(\phi\left(t_{2}\right)\right)\right)-g\left(t_{1}, x\left(\psi\left(t_{1}\right)\right)\right) I^{\alpha} f\left(t_{2}, x\left(\phi\left(t_{2}\right)\right)\right) \\
\leq & a\left(t_{2}\right)-a\left(t_{1}\right)+\left[g\left(t_{2}, x\left(\psi\left(t_{2}\right)\right)\right)-g\left(t_{1}, x\left(\psi\left(t_{1}\right)\right)\right)\right] I^{\alpha} f\left(t_{2}, x\left(\phi\left(t_{2}\right)\right)\right) \\
& \quad+g\left(t_{1}, x\left(\psi\left(t_{1}\right)\right)\right)\left[I^{\alpha} f\left(t_{2}, x\left(\phi\left(t_{2}\right)\right)\right)-I^{\alpha} f\left(t_{1}, x\left(\phi\left(t_{1}\right)\right)\right)\right],
\end{aligned}
$$

but

$$
\begin{gathered}
I^{\alpha} f\left(t_{2}, x\left(\phi\left(t_{2}\right)\right)\right)-I^{\alpha} f\left(t_{1}, x\left(\phi\left(t_{1}\right)\right)\right)=\int_{0}^{t_{1}} \frac{\left(t_{2}-s\right)^{\alpha-1}}{\Gamma(\alpha)} f(s, x(\phi(s))) d s \\
\quad+\int_{t_{1}}^{t_{2}} \frac{\left(t_{2}-s\right)^{\alpha-1}}{\Gamma(\alpha)} f(s, x(\phi(s))) d s-\int_{0}^{t_{1}} \frac{\left(t_{1}-s\right)^{\alpha-1}}{\Gamma(\alpha)} f(s, x(\phi(s))) d s \\
\leq \int_{0}^{t_{1}} \frac{\left(t_{1}-s\right)^{\alpha-1}}{\Gamma(\alpha)} f(s, x(\phi(s))) d s+\int_{t_{1}}^{t_{2}} \frac{\left(t_{2}-s\right)^{\alpha-1}}{\Gamma(\alpha)} f(s, x(\phi(s))) d s \\
-\int_{0}^{t_{1}} \frac{\left(t_{1}-s\right)^{\alpha-1}}{\Gamma(\alpha)} f(s, x(\phi(s))) d s=\int_{t_{1}}^{t_{2}} \frac{\left(t_{2}-s\right)^{\alpha-1}}{\Gamma(\alpha)} f(s, x(\phi(s))) d s .
\end{gathered}
$$

Then

$$
\begin{gathered}
\left|I^{\alpha} f\left(t_{2}, x\left(\phi\left(t_{2}\right)\right)\right)-I^{\alpha} f\left(t_{1}, x\left(\phi\left(t_{1}\right)\right)\right)\right| \leq I_{t_{1}}^{\alpha}\left|f\left(t_{2}, x\left(\phi\left(t_{2}\right)\right)\right)\right| \\
\leq I_{t_{1}}^{\alpha} m\left(t_{2}\right)+b I_{t_{1}}^{\alpha}\left|x\left(\phi\left(t_{2}\right)\right)\right| \leq I_{t_{1}}^{\alpha-\beta} I_{t_{1}}^{\beta} m\left(t_{2}\right)+b I_{t_{1}}^{\alpha}\left|x\left(\phi\left(t_{2}\right)\right)\right| \\
\leq k_{3} \frac{\left(t_{2}-t_{1}\right)^{\alpha-\beta}}{\Gamma(\alpha-\beta+1)}+b r \frac{\left(t_{2}-t_{1}\right)^{\alpha}}{\Gamma(\alpha+1)} .
\end{gathered}
$$

Then we get

$$
\begin{gathered}
\left|(T x)\left(t_{2}\right)-(T x)\left(t_{1}\right)\right| \\
\leq\left|a\left(t_{2}\right)-a\left(t_{1}\right)\right|+\left[l_{1}\left|t_{2}-t_{1}\right|+l_{2}\left|x\left(\psi\left(t_{2}\right)\right)-x\left(\psi\left(t_{1}\right)\right)\right|\right] \\
I^{\alpha}\left|f\left(t_{2}, x\left(\phi\left(t_{2}\right)\right)\right)\right| \\
+\left|g\left(t_{1}, x\left(\psi\left(t_{1}\right)\right)\right)\right|\left(k_{3} \frac{\left(t_{2}-t_{1}\right)^{\alpha-\beta}}{\Gamma(\alpha-\beta+1)}+b r \frac{\left(t_{2}-t_{1}\right)^{\alpha}}{\Gamma(\alpha+1)}\right)
\end{gathered}
$$

i.e.,

$$
\begin{gathered}
\left|(T x)\left(t_{2}\right)-(T x)\left(t_{1}\right)\right| \\
\leq\left|a\left(t_{2}\right)-a\left(t_{1}\right)\right|+\left[l_{1}\left|t_{2}-t_{1}\right|+l_{2}\left|x\left(t_{2}\right)-x\left(t_{1}\right)\right|\right] \\
I^{\alpha}\left(m\left(t_{2}\right)+b\left|x\left(\phi\left(t_{2}\right)\right)\right|\right)
\end{gathered}
$$




$$
\begin{gathered}
+k_{2} k_{3} \frac{\left(t_{2}-t_{1}\right)^{\alpha-\beta}}{\Gamma(\alpha-\beta+1)}+k_{2} b r \frac{\left(t_{2}-t_{1}\right)^{\alpha}}{\Gamma(\alpha+1)} \\
\leq\left|a\left(t_{2}\right)-a\left(t_{1}\right)\right|+\frac{k_{3}}{\Gamma(\alpha-\beta+1)}\left[l_{1}\left|t_{2}-t_{1}\right|+l_{2}\left|x\left(t_{2}\right)-x\left(t_{1}\right)\right|\right] \\
+\frac{b r}{\Gamma(\alpha+1)}\left[l_{1}\left|t_{2}-t_{1}\right|+l_{2}\left|x\left(t_{2}\right)-x\left(t_{1}\right)\right|\right]+\frac{k_{2} k_{3}}{\Gamma(\alpha-\beta+1)}\left(t_{2}-t_{1}\right)^{\alpha-\beta} \\
+\frac{k_{2} b r}{\Gamma(\alpha+1)}\left(t_{2}-t_{1}\right)^{\alpha} \rightarrow 0 \quad \text { as } t_{2} \rightarrow t_{1} .
\end{gathered}
$$

This means that the functions of $T S_{r}$ are equi-continuous on $I$. Then by the ArzelaAscoli Theorem [9] the closure of $T S_{r}$ is compact .

It is clear that the set $S_{r}$ is nonempty, bounded, closed and convex.

Assumptions (ii) and (iv) imply that $T: S_{r} \rightarrow C(I)$ is a continuous operator in $x$.

Since all conditions of the Schauder fixed-point theorem hold, then $T$ has a fixed point in $S_{r}$.

\section{Special cases}

Corollary 1. Let the assumptions of Theorem 2 be satisfied (with $\psi(t)=\phi(t)=t)$, then the fractional-order quadratic integral equation

$$
x(t)=a(t)+g(t, x(t)) \int_{0}^{t} \frac{(t-s)^{\alpha-1}}{\Gamma(\alpha)} f(s, x(s)) d s
$$

has at least one solution $x \in C$.

Corollary 2. Let the assumptions of Theorem 2 be satisfied (with $g(t, x)=1$ ), then the fractional-order integral equation

$$
x(t)=a(t)+\int_{0}^{t} \frac{(t-s)^{\alpha-1}}{\Gamma(\alpha)} f(s, x(\phi(s))) d s
$$

has at least one solution $x \in C$.

Letting $b=0$ and $\phi(t)=t$ in Corollary 2, we obtain the same result as was proved in [18].

Now letting $\alpha, \beta \rightarrow 1$, we obtain

Corollary 3. Let the assumptions of Theorem 2 be satisfied (with $g(t, x)=1, a(t)=$ $x_{0}$ and letting $\alpha, \beta \rightarrow 1$ ), then the integral equation

$$
x(t)=x_{0}+\int_{0}^{t} f(s, x(\phi(s))) d s .
$$

has at least one solution $x \in C$ which is equivalent to the initial value problem (1.3).

Letting $b=0$ and $\phi(t)=t$ in Corollary 3 we obtain the Carathéodory Theorem (proved in [9]). 


\section{FRACTIONAL ORDER FUNCTIONAL DIFFERENTIAL EQUATIONS}

For the initial value problem of the nonlinear fractional-order differential equation (1.2) we have the following theorem.

Theorem 3. Let the assumptions of Theorem 2 be satisfied (with $a(t)=0$ and $g(t, x(t))=1)$, then the Cauchy type problem (1.2) has at least one solution $x \in C$.

Proof. Integrating (1.2) we obtain the integral equation

$$
x(t)=\int_{0}^{t} \frac{(t-s)^{\alpha-1}}{\Gamma(\alpha)} f(s, x(\phi(s))) d s, \quad t \in I
$$

which by Theorem 2 has the desired solution.

Operating with ${ }_{R} D^{\alpha}$ on (5.1) we obtain the initial value problem (1.2). So the equivalence between the initial value problem(1.2) and the integral equation (5.1) is proved and then the results follow from Theorem 2.

\section{MAXimAL AND Minimal SOLUTIONS}

Definition 3 (see [21]). Let $q(t)$ be a solution of (1.1) Then $q(t)$ is said to be a maximal solution of (1.1) if every solution of (1.1) on $I$ satisfies the inequality $x(t) \leq q(t), t \in I$. A minimal solution $s(t)$ can be defined in a similar way by reversing the above inequality i.e. $x(t) \geq s(t), t \in I$.

We need the following lemma to prove the existence of maximal and minimal solutions of (1.1).

Lemma 1. Let $g(t, x), f(t, x)$ satisfy the assumptions in Theorem 2 and let $x(t), y(t)$ be continuous functions on I satisfying

$$
\begin{aligned}
& x(t) \leq a(t)+g(t, x(\psi(t))) I^{\alpha} f(t, x(\phi(t))) \\
& y(t) \geq a(t)+g(t, y(\psi(t))) I^{\alpha} f(t, y(\phi(t)))
\end{aligned}
$$

where one of them is strict.

Suppose $f(t, x)$ is nondecreasing function in $x$. Then

$$
x(t)<y(t) .
$$

Proof. Let the conclusion (6.1) be false; then there exists $t_{1}$ such that

$$
x\left(t_{1}\right)=y\left(t_{1}\right), \quad t_{1}>0
$$

and

$$
x(t)<y(t), \quad 0<t<t_{1} .
$$


From the monotonicity of the function $f$ in $x$, we get

$$
\begin{aligned}
x\left(t_{1}\right) & \leq a\left(t_{1}\right)+g\left(t_{1}, x\left(\psi\left(t_{1}\right)\right)\right) I^{\alpha} f\left(t_{1}, x\left(\phi\left(t_{1}\right)\right)\right) \\
& =a\left(t_{1}\right)+g\left(t_{1}, x\left(\psi\left(t_{1}\right)\right)\right) \int_{0}^{t_{1}} \frac{\left(t_{1}-s\right)^{\alpha-1}}{\Gamma(\alpha)} f(s, x(\phi(s))) d s \\
& <a\left(t_{1}\right)+g\left(t_{1}, y\left(\psi\left(t_{1}\right)\right)\right) \int_{0}^{t_{1}} \frac{\left(t_{1}-s\right)^{\alpha-1}}{\Gamma(\alpha)} f(s, y(\phi(s))) d s \\
& <y\left(t_{1}\right) .
\end{aligned}
$$

This contradicts the fact that $x\left(t_{1}\right)=y\left(t_{1}\right)$; then

$$
x(t)<y(t) .
$$

Theorem 4. Let the assumptions of Theorem 2 be satisfied. Furthermore, if $f(t, x)$ is nondecreasing functions in $x$, then there exist maximal and minimal solutions of (1.1).

Proof. Firstly, we shall prove the existence of maximal solution of (1.1). Let $\epsilon>$ 0 be given. Now consider the fractional-order quadratic functional integral equation

$$
x_{\epsilon}(t)=a(t)+g_{\epsilon}\left(t, x_{\epsilon}(\psi(t))\right) I^{\alpha} f_{\epsilon}\left(t, x_{\epsilon}(\phi(t))\right),
$$

where

$$
f_{\epsilon}\left(t, x_{\epsilon}(\phi(t))\right)=f\left(t, x_{\epsilon}(\phi(t))\right)+\epsilon
$$

and

$$
g_{\epsilon}\left(t, x_{\epsilon}(\psi(t))\right)=g\left(t, x_{\epsilon}(\psi(t))\right)+\epsilon .
$$

Clearly the functions $f_{\epsilon}\left(t, x_{\epsilon}\right)$ and $g_{\epsilon}\left(t, x_{\epsilon}\right)$ satisfy assumptions (ii), (iv) and

$$
\left|g_{\epsilon}\left(t, x_{\epsilon}\right)\right| \leq M+\epsilon=M^{\prime} \text {. }
$$

$$
\left|f_{\epsilon}\left(t, x_{\epsilon}\right)\right| \leq m(t)+\epsilon+b|x|=m^{\prime}(t)+b|x| .
$$

Therefore, equation (6.2) has a continuous solution $x_{\epsilon}(t)$ according to Theorem 2. Let $\epsilon_{1}$ and $\epsilon_{2}$ be such that $0<\epsilon_{2}<\epsilon_{1}<\epsilon$. Then

$$
\begin{gathered}
x_{\epsilon_{1}}(t)=a(t)+g_{\epsilon_{1}}\left(t, x_{\epsilon_{1}}(\psi(t))\right) I^{\alpha} f_{\epsilon_{1}}\left(t, x_{\epsilon_{1}}(\phi(t))\right), \\
x_{\epsilon_{1}}(t)=a(t)+\left(g\left(t, x_{\epsilon_{1}}(\psi(t))\right)+\epsilon_{1}\right) I^{\alpha}\left(f\left(t, x_{\epsilon_{1}}(\phi(t))\right)+\epsilon_{1}\right), \\
>a(t)+\left(g\left(t, x_{\epsilon_{1}}(\psi(t))\right)+\epsilon_{2}\right) I^{\alpha}\left(f\left(t, x_{\epsilon_{1}}(\phi(t))\right)+\epsilon_{2}\right), \\
x_{\epsilon_{2}}(t)=a(t)+\left(g\left(t, x_{\epsilon_{2}}(\psi(t))\right)+\epsilon_{2}\right) I^{\alpha}\left(f\left(t, x_{\epsilon_{2}}(\phi(t))\right)+\epsilon_{2}\right) .
\end{gathered}
$$


Applying Lemma 1, then (6.3) and (6.4) imply

$$
x_{\epsilon_{2}}(t)<x_{\epsilon_{1}}(t) \quad \text { for } t \in I .
$$

As shown before in the proof of Theorem 2, the family of functions $x_{\epsilon}(t)$ defined by (6.2) is uniformly bounded and of equi-continuous functions. Hence by the ArzelaAscoli Theorem, there exists a decreasing sequence $\epsilon_{n}$ such that $\epsilon_{n} \rightarrow 0$ as $n \rightarrow$ $\infty$, and $\lim _{n \rightarrow \infty} x_{\epsilon_{n}}(t)$ exists uniformly in $I$. We denote this limit by $q(t)$. From the continuity of the functions $f_{\epsilon_{n}}$ and $g_{\epsilon_{n}}$ in the second argument, we get

$$
q(t)=\lim _{n \rightarrow \infty} x_{\epsilon_{n}}(t)=a(t)+g(t, q(\psi(t))) I^{\alpha} f(t, q(\phi(t)))
$$

which proves that $q(t)$ is a solution of (1.1).

Finally, we shall show that $q(t)$ is maximal solution of (1.1). To do this, let $x(t)$ be any solution of (1.1). Then

$$
\begin{aligned}
& x_{\epsilon}(t)=a(t)+g_{\epsilon}\left(t, x_{\epsilon}(\psi(t))\right) I^{\alpha} f_{\epsilon}\left(t, x_{\epsilon}(\phi(t))\right) \\
& >a(t)+g\left(t, x_{\epsilon}(\psi(t))\right) I^{\alpha} f\left(t, x_{\epsilon}(\phi(t))\right) .
\end{aligned}
$$

and

$$
x(t)=a(t)+g(t, x(\psi(t))) I^{\alpha} f(t, x(\phi(t))) .
$$

Applying Lemma 1, we get

$$
x_{\epsilon}(t)>x(t) \quad \text { for } t \in I .
$$

from the uniqueness of the maximal solution (see [21], [24]), it is clear that $x_{\epsilon}(t)$ tends to $q(t)$ uniformly in $t \in I$ as $\epsilon \rightarrow 0$.

In a similar way we can prove that there exists a minimal solution of (1.1).

\section{ACKNOWLEDGMENT:}

The authors are thankful to the referee for the time taken to review this paper and for the remarks and suggestions that helped improve the quality of this paper.

\section{REFERENCES}

[1] I. K. Argyros, "Quadratic equations and applications to Chandrasekhar's and related equations," Bull. Aust. Math. Soc., vol. 32, pp. 275-292, 1985.

[2] I. K. Argyros, "On a class of quadratic integral equations with perturbation," Funct. Approximatio, Comment. Math., vol. 20, pp. 51-63, 1992.

[3] J. Banaś, J. Caballero, J. Rocha, and K. Sadarangani, "Monotonic solutions of a class of quadratic integral equations of Volterra type," Comput. Math. Appl., vol. 49, no. 5-6, pp. 943-952, 2005.

[4] J. Banaś, J. R. Martin, and K. Sadarangani, "On solutions of a quadratic integral equation of Hammerstein type," Math. Comput. Modelling, vol. 43, no. 1-2, pp. 97-104, 2006.

[5] J. Banaś and A. Martinon, "Monotonic solutions of a quadratic integral equation of Volterra type," Comput. Math. Appl., vol. 47, no. 2-3, pp. 271-279, 2004.

[6] J. Banaś and B. Rzepka, "Nondecreasing solutions of a quadratic singular Volterra integral equation," Math. Comput. Modelling, vol. 49, no. 3-4, pp. 488-496, 2009. 
[7] J. Banaś, M. Lecko, and W. G. El-Sayed, "Existence theorems for some quadratic integral equations," J. Math. Anal. Appl., vol. 222, no. 1, pp. 276-285, 1998.

[8] J. Banaś and B. Rzepka, "Monotonic solutions of a quadratic integral equation of fractional order," J. Math. Anal. Appl., vol. 332, no. 2, pp. 1371-1379, 2007.

[9] R. F. Curtain and A. J. Pritchard, Functional analysis in modern applied mathematics, ser. Mathematics in Science and Engineering. London, New York: Academic Press (Harcourt Brace Jovanovich, Publishers), 1977, vol. 132.

[10] M. A. Darwish, "On monotonic solutions of a singular quadratic integral equation with supremum," Dyn. Syst. Appl., vol. 17, no. 3-4, pp. 539-549, 2008.

[11] M. M. El Borai, W. G. El-Sayed, and M. I. Abbas, "Monotonic solutions of a class of quadratic singular integral equations of Volterra type," Int. J. Contemp. Math. Sci., vol. 2, no. 1-4, pp. 89$102,2007$.

[12] A. M. A. El-Sayed and H. H. G. Hashem, "Carathéodory type theorem for a nonlinear quadratic integral equation,” Math. Sci. Res. J., vol. 12, no. 4, pp. 88-95, 2008.

[13] A. M. A. El-Sayed and H. H. G. Hashem, "Integrable and continuous solutions of a nonlinear quadratic integral equation,” Electron. J. Qual. Theory Differ. Equ., vol. 2008, p. 10, 2008.

[14] A. M. A. El-Sayed and H. H. G. Hashem, "Integrable solutions for quadratic Hammerstein and quadratic Urysohn functional integral equations," Commentat. Math., vol. 48, no. 2, pp. 199-207, 2008.

[15] A. M. A. El-Sayed and H. H. G. Hashem, "Monotonic solutions of functional integral and differential equations of fractional order," Electron. J. Qual. Theory Differ. Equ., vol. 2009, pp. 1-8, 2009.

[16] A. M. A. El-Sayed and H. H. G. Hashem, "Monotonic positive solution of a nonlinear quadratic functional integral equation," Appl. Math. Comput., vol. 216, no. 9, pp. 2576-2580, 2010.

[17] A. M. A. El-Sayed, H. H. G. Hashem, and E. A. A. Ziada, "Picard and Adomian methods for quadratic integral equation," Comput. Appl. Math., vol. 29, no. 3, pp. 447-463, 2010.

[18] A. M. A. El-Sayed, F. M. Gaafar, and H. H. G. Hashem, "On the maximal and minimal solutions of arbitrary-orders nonlinear functional integral and differential equations," Math. Sci. Res. J., vol. 8, no. 11, pp. 336-348, 2004

[19] W. G. El-Sayed and B. Rzepka, "Nondecreasing solutions of a quadratic integral equation of Urysohn type," Comput. Math. Appl., vol. 51, no. 6-7, pp. 1065-1074, 2006.

[20] A. A. Kilbas, H. M. Srivastava, and J. J. Trujillo, Theory and applications of fractional differential equations, ser. North-Holland Mathematics Studies. Amsterdam: Elsevier, 2006, vol. 204.

[21] V. Lakshmikantham and S. Leela, Differential and integral inequalities. Theory and applications. Vol. I: Ordinary differential equations, ser. Mathematics in Science and Engineering. New YorkLondon: Academic Press, 1969, vol. 55.

[22] K. S. Miller and B. Ross, An introduction to the fractional calculus and fractional differential equations. New York: John Wiley \& Sons, Inc., 1993.

[23] I. Podlubny, Fractional differential equations. An introduction to fractional derivatives, fractional differential equations, to methods of their solution and some of their applications, ser. Mathematics in Science and Engineering. San Diego, CA: Academic Press, 1999, vol. 198.

[24] M. R. M. Rao, Ordinary differential equations. Theory and applications. New Delhi-Madras: Affiliated East-West Press Pvt Ltd., 1980.

[25] H. A. H. Salem, "On the quadratic integral equations and their applications," Comput. Math. Appl., vol. 62, no. 8, pp. 2931-2943, 2011.

[26] S. G. Samko, A. A. Kilbas, and O. I. Marichev, Integrals and derivatives of fractional order and some of their applications. Minsk: Nauka i Tekhnika, 1987. 
Authors' addresses

A. M. A. El-Sayed

Faculty of Science, Alexandria University, Math. Department, Shatby St., Alexandria, Egypt

E-mail address: amasayed@hotmail.com

H. H. G. Hashem

Faculty of Science, Alexandria University, Math. Department, Shatby St., Alexandria, Egypt

Current address: Qassim University, College of Science, Math. Department, P. O. Box 6644, Buraidah 81999, Saudi Arabia

E-mail address: hendhghashem@yahoo.com / hindhghashemegmail.com 